\title{
Desenvolvimento de um Sistema de Computação para Análise de Estruturas Planas
}

\author{
Luanna Lopes Lobato', Thiago Jabur Bittar'1, Ivo Palheta Mendes ${ }^{1}$, Wanderlei \\ Malaquias Pereira Junior² \\ ${ }^{1}$ Departamento de Ciência da Computação - Universidade Federal de Catalão (UFCAT) \\ - Catalão, GO - Brasil \\ ${ }^{2}$ Departamento de Engenharia Civil - Universidade Federal de Catalão (UFCAT) - \\ Catalão, GO - Brasil \\ \{luannalobato, thiagojabur, wanderlei_junior\}@ufg.br
}

\begin{abstract}
Software Engineering has been present in different researches on the computer systems development is required to optimize, bring quality and safety to tasks. This paper presents the study on CAD systems and reports the software implementation to perform analysis of flat structures such as beams, trusses and gantries, providing the education on Civil Engineering course. For this, the recommendations imposed in Software Engineering were considered, together with the Civil Engineering peculiarities, presenting an interface for routines implementation. A Case Study was applied to collect data, which resulted in improvements to apply in the system.
\end{abstract}

Resumo. A Engenharia de Software tem estado presente em diferentes pesquisas em que é requerido o desenvolvimento de sistemas computacionais para otimizar, trazer qualidade e segurança nas tarefas. Neste artigo é apresentado o estudo sobre os aspectos dos sistemas CAD e a implementação de um sistema para realizar análise de estruturas planas como vigas, treliças e pórticos, voltado para o ensino no curso de Engenharia Civil. Para tanto, foram consideradas as recomendações impostas na Engenharia de Software, juntamente com as peculiaridades da Engenharia Civil, disponibilizando uma interface para a implantação de rotinas. Um estudo de caso foi aplicado para a coleta de dados, o que resultou no levantamento de melhorias do sistema.

\section{Introdução}

Uma categoria de software muito utilizada nas grandes áreas como engenharia, geologia e design é de sistemas de Computer-Aided Design (CAD) ou Desenho Assistido por Computador [Sarcar, Rao e Narayan, 2008]. O sistema CAD tem se tornado uma potente ferramenta para a indústria moderna, permitindo a modelagem de produtos com formas complexas, realização de análises de formas geométricas planas (linhas, curvas, polígonos), tridimensionais (cubos, esferas), para auxiliar a manufatura e análises de interferências entre peças e conjuntos. É possível utilizar-se dos objetos construídos para a representação de entidades, como representar elementos de engenharia estrutural referentes a barras, vigas, treliças e pórticos [Korpyljov e Tkachenko, 2013].

Seguindo essa vertente, neste trabalho é descrito o desenvolvimento e avaliação de um sistema computacional, com ambiente CAD bidimensional, nomeado EngPack 
Analysis, para apoiar a modelagem e a análise de estruturas planas, de maneira gratuita aos usuários. Esta pesquisa tem a colaboração de pesquisadores da Universidade Federal de Catalão (UFCAT), dos Departamentos de Ciência da Computação (CC), área de Engenharia de Software (ES), e Engenharia Civil (EC).

Ao final da implementação do sistema foi aplicado um estudo de caso para coletar as impressões dos usuários, para análise de verificação e validação do sistema. Assim, buscou-se por meio da análise dos resultados, verificar a qualidade do sistema referente aos requisitos imprescindíveis para sistemas desta categoria, confirmar a possibilidade de otimização de tarefas e segurança sobre os dados processados, uma vez que cálculos estruturais devem ser considerados. Adicionalmente, tentou-se com esta pesquisa, contribuir também para os alunos do curso de CC que estudaram os conceitos nas disciplinas de ES1, ES2 e Fábrica de Software (FS), referente aos aspectos técnicos acerca do uso das metodologias e técnicas de desenvolvimento de software, mais especificamente utilizando-se de algumas lições encorajadas pelas metodologias ágeis. Assim, os alunos puderam aplicar os conceitos de forma prática durante 0 desenvolvimento do sistema computacional, a ser utilizado por diferentes usuários.

O restante deste artigo está organizado do seguinte modo: na Seção 1.1 são apresentados aspectos de motivação e objetivos da pesquisa. Na Seção 1.2 tem-se as contribuições do trabalho. Na Seção 2 são apresentados os passos da metodologia de pesquisa adotada juntamente com os trabalhos relacionados. Na Seção 3 tem-se a área de estudo apresentada incluindo a revisão bibliográfica da base conceitual do trabalho. Já na Seção 4 tem-se a apresentação de itens arquiteturais e de algoritmos do desenvolvimento do sistema. Por fim, na Seção 5 tem-se as discussões e resultados obtidos.

\subsection{Motivação e Objetivos}

Muitos dos sistemas que proveem suporte às disciplinas da EC, mais especificamente àqueles que envolvem análise estrutural, tem alto valor para aquisição de licença e quando disponibilizam versões gratuitas essas são limitadas [Bizello e Ruschel, 2011]. Ainda, alguns softwares de análise estrutural possuem limitações de compatibilidade com diferentes Sistemas Operacionais (SO), o que restringe o usuário a específicas plataformas na qual pode ser executado, o que torna necessária a aquisição das licenças caras para uso completo das funcionalidades.

Dada essas restrições, justifica-se essa pesquisa pelo desenvolvimento de um sistema multiplataforma que, por meio do uso de recursos computacionais, pode ser utilizado para o ensino na área de engenharia estrutural, modelagem e análise de estruturas planas. Este foi implementado de forma modularizada, o que possibilita sua evolução para análise de outros tipos de estruturas, possibilitando contribuições futuras às áreas envolvidas.

Ainda, a motivação foi calcada na necessidade de se ter um software gratuito, que atenda à demanda do departamento de EC da UFCAT, nas disciplinas da área de engenharia estrutural, na modelagem e análise de estruturas planas. Por se tratar de um sistema gratuito, espera-se também que o mesmo possa ser utilizado em diferentes Instituições, como suporte ao ensino e aprendizado.

Assim, teve-se como objetivo principal a pesquisa e construção do sistema computacional de análise de estruturas a ser aplicado na engenharia. Para atingir esse 
objetivo, definiu-se alguns específicos, que foram seguidos nesta pesquisa: 1) Revisão bibliográfica acerca das metodologias de desenvolvimento de software, com foco nas ágeis XP e framework de gerência SCRUM [Sommerville, 2011]; 2) Revisão bibliográfica sobre sistemas CAD; 3) Pesquisa e inspeção dos sistemas CAD para análise de estruturas; 4) Implementação de interface gráfica condizente com os padrões firmados no mercado, para esse tipo de sistema; 5) Implantação das rotinas de cálculo e análise em estruturas planas; 6) Realização de testes e avaliações de usabilidade com os usuários finais.

\subsection{Contribuições do Trabalho}

Uma vez que o EngPack Analysis seguiu padrões de sistemas CAD consolidados, acredita-se que a curva de aprendizado para sua utilização, no meio acadêmico, seja reduzida. Assim, deseja-se que os usuários, inicialmente os alunos da EC, possam ter boa familiaridade com o mesmo, tendo agilidade na realização das atividades oferecidas por meio das funcionalidades. É importante ressaltar a funcionalidade que possibilita que os usuários implementem rotinas de cálculos em análise estrutural, utilizando as interfaces de implementação desenvolvidas.

No que tange os aspectos da ES foram utilizadas, para o desenvolvimento do EngPack Analysis, metodologias de desenvolvimento de software e padrões de arquitetura, bem como ferramentas para codificação de sistema e testes. Assim, alguns conceitos são brevemente apresentados. Ainda, são abordados conceitos e fundamentos básicos da engenharia estrutural e análise de estruturas, os quais são essenciais para o entendimento da modelagem do sistema desenvolvido. Por fim, é mostrado o sistema computacional desenvolvido, seguido de sua avaliação e resultados.

\section{Metodologia de Pesquisa}

A metodologia de pesquisa utilizada é composta por duas fases: a fase de concepção e a de desenvolvimento e avaliação, adaptada de Lobato [2012]. Na primeira fase, os conceitos são estudados, as necessidades pontuadas e as restrições identificadas, para obtenção de informações e constituição da base de dados de conhecimento. A segunda fase refere-se à construção do sistema, para consequente avaliação do mesmo.

Assim, a fase de Concepção foi dividida em: Revisão da Literatura, Análise de Trabalhos Relacionados, Análise de Software Existentes, Base de conhecimento e Definição do Escopo do Sistema. Estas foram necessárias para formar a base de conhecimento para proposta e desenvolvimento do sistema, fortalecer o conhecimento sobre sistemas CAD, relacionando os métodos e técnicas aplicáveis.

A Revisão da Literatura foi ad-hoc, com foco em desenvolvimento de sistemas e análise de estruturas, buscando identificar gaps nas áreas e quais métodos e técnicas são comumente utilizados, bem como aprender sobre os conceitos da engenharia estrutural, a fim de abstrair os principais elementos estruturais, os quais foram necessários para a modelagem das estruturas analisadas pelo sistema desenvolvido.

Seguindo, fez-se a revisão dos trabalhos relacionados, que abordou estudos sobre o desenvolvimento de sistemas que apoiem disciplinas de engenharia estrutural e análise de estruturas. Nesta fase da pesquisa foram pontuados padrões de funcionamento e comportamento de ferramentas de edição em ambientes CAD. Por fim, foram feitos estudos detalhados sobre os sistemas de análise estrutural disponíveis, verificando suas 
características essenciais. Os padrões observados foram seguidos para que o EngPack Analysis fosse facilmente aceito por usuários que tiveram algum contato com os sistemas disponíveis no mercado e no meio acadêmico.

Por meio das etapas descritas teve-se a base de conhecimento acerca das características e padrões de sistemas CAD para análise de estruturas, o que validou os requisitos iniciais e possibilitou delimitar o escopo para o sistema desenvolvido.

A segunda fase, Desenvolvimento e Avaliação, é referente à construção e validação do EngPack Analysis. Nesta foi empregada a metodologia ágil para desenvolvimento de software. Inicialmente, teve-se a etapa de Engenharia de Requisitos, que denotou em estudos essenciais para a elicitação, validação e especificação de requisitos funcionais (RF) e não funcionais (RNF) acerca das características e funcionalidades do EngPack Analysis.

Em seguida, teve-se o Projeto e Implementação do sistema, utilizando-se de práticas de desenvolvimento ágil aprendidas na disciplina de ES. A próxima etapa refere-se à Avaliação do Sistema, onde foi verificado se o os requisitos especificados são atendidos, sendo realizada junto aos colaboradores do projeto, se verificado mudanças essenciais estas eram feitas. Uma vez que o sistema cumpre corretamente o que foi especificado, foi executada a etapa de Testes. Após o sistema validado e verificado que não há mais modificações essenciais, ele foi considerado estável, sendo finalizada a etapa de entrega.

Assim, buscou-se com a execução desta metodologia de pesquisa garantir qualidade do sistema, segurança sobre os resultados gerados, precisão e acurácia sobre as operações realizadas.

\subsection{Trabalhos Relacionados}

Esta pesquisa é parte de um projeto da UFCAT, entre os departamentos de CC e EC, sendo assim, em que alguns trabalhos envolvendo a EC foram parceiros a esta pesquisa.

Neste sentido, pode-se ressaltar o trabalho de Dutra [2018] que tem por objetivo o desenvolvimento das rotinas que efetuam os cálculos para a análise de vigas através dos métodos das forças e dos deslocamentos, onde usa-se da análise matricial para obtenção de esforços em estruturas de vigas planas. Neste estudo foi observada a necessidade de uma ferramenta computacional para o cálculo de esforço em vigas, sendo que os resultados obtidos por meio dos cálculos de esforços foram transferidos para o protótipo da interface gráfica do EngPack Analysis, que ainda estava em estágio inicial. Atualmente, o sistema fornece uma interface gráfica estável para o uso da análise matricial, considerando o uso de uma das rotinas implantadas em Dutra [2018]. Assim, pode-se obter alguns resultados por meio de análise estrutural de vigas isostática e hiperestáticas utilizando o método de rigidez direta.

Adicionalmente, Dutra [2018] utiliza protótipos iniciais do sistema EngPack Analysis para o desenvolvimento e aplicação de rotinas para cálculos de análise de treliças planas. Neste é ressaltada a necessidade por um sistema de análise estrutural, mas o trabalho mantem o foco no desenvolvimento das rotinas, evitando a abordagem sobre desenvolvimento de software. Este também é um projeto parceiro, colaborando para composição do módulo de análise estrutural definido no sistema EngPack Analysis. 
O FTOOL [Martha, 2012] é um software conhecido no mercado e no meio acadêmico, que possui interface gráfica interativa para o ensino de comportamentos estruturais. Entretanto, ele não aborda conceitos sobre o sistema computacional, diferentemente desta pesquisa, que foca no processo de desenvolvimento do software.

Mais próximo ao desenvolvimento de software, tem-se o trabalho de Longo [2015], que descreve o desenvolvimento de um sistema portátil para plataforma Android e disponibiliza ferramentas para a análise de estruturas. O aplicativo é uma alternativa de programa de análise estrutural para estudo simplificado de estruturas em sala de aula. A validação dos resultados feita de forma comparativa com os resultados do FTOOL.

Já Viegas e Vargas [2015] desenvolveram um software que apresenta, em tempo real, as ações dos carregamentos em uma viga, tendo como resultado as reações nos apoios, esforço cortante e momento fletor, assim como os diagramas. Este foi desenvolvido para ajudar em aulas que envolvam apenas a análise de vigas e possui interface limitada no processo de modelagem e edição das vigas, que só podem ser manipuladas por meio das janelas de inserção e edição.

Em contrapartida, Kaefer [2000] apresenta uma ferramenta gráfica interativa para a modelagem e dimensionamento de pórticos de planos de concreto armado. Os resultados são validados com os cálculos de análise de Soler, utilizando uma formulação que permite que as vigas sejam submetidas a grandes deslocamentos, rotações de deformações.

Os sistemas CAD fornecem soluções também em outras áreas e alguns trabalhos, mesmo que de forma limitada, contribuíram para a condução deste, mas não foram especificados por não envolver as áreas de estudo. Na maioria dos trabalhos relacionados o foco está na implementação das rotinas de cálculo para a execução da análise das estruturas, o que difere deste que foca no processo de desenvolvimento do sistema computacional para produzir uma ferramenta para análise de estruturas.

\section{3. Área de Estudo}

Dada a interdisciplinaridade entre a ES e EC para a implementação de um sistema de computação aplicados a engenharia, nas seções que seguem são descritos, brevemente, os conceitos utilizados para desenvolvimento desta pesquisa.

\subsection{Aspectos da Engenharia de Software}

Sommerville [2019] descreve o projeto de arquitetura como uma etapa importante do processo de software, que antecede a implementação, gerando como resultado um modelo de arquitetura que descreve como o sistema está organizado em um conjunto de componentes de comunicação e sua estrutura geral.

A abordagem de desenvolvimento empregada nesta pesquisa foi a ágil, assim o estágio inicial do processo de desenvolvimento focou no estabelecimento de uma arquitetura global do sistema. É importante salientar que as noções de separação e independência são fundamentais para o projeto de arquitetura, porque permitem que alterações sejam facilmente localizadas. Para o levantamento dos requisitos foram realizadas entrevistas com clientes e desenvolvedores, definindo a descrição de cenários e executando casos de uso - os quais não são detalhados devido à limitação de páginas. 
O padrão de arquitetura utilizado no projeto do EngPack Analysis foi o ModeloVisão-Controlador (MVC), que separa a apresentação e a interação dos dados do sistema, estruturado em três componentes lógicos que interagem entre si. O padrão MVC permite que os dados sejam alterados de forma independente de sua representação e vice-versa [Luciano e Alves, 2011].

Para a implementação do sistema utilizou-se alguns padrões de projeto, tais como o Singleton (Criacional), o Composite (Estrutural), o Observer e o Command (Comportamentais). O padrão Singleton garante a existência de apenas uma instância de uma classe, mantendo um ponto global de acesso ao seu objeto [Gamma et al., 1995].

\subsection{Aspectos da Engenharia Estrutural}

Além dos conceitos de ES, fez-se necessário abordar conceitos acerca da aplicação do sistema, ou seja, sobre a sua finalidade para análise de estruturas planas, uma vez que envolvidos no projeto não detinham desse conhecimento.

A engenharia estrutural é onde são criadas as estruturas fundamentais que dão forma às construções edificadas, assim, é preciso entender e calcular a estabilidade, força e rigidez de estruturas construídas e estruturas não construídas [Soriano, 2013]. A teoria da engenharia estrutural é baseada em leis físicas.

A análise estrutural emprega os campos da mecânica aplicada, ciência dos materiais e matemática para calcular as deformações de uma estrutura, forças internas, tensões, reações de apoio, acelerações e estabilidade [Soriano, 2013]. Os resultados da análise são usados para verificar a adequação de uma estrutura ao uso, frequentemente evitando testes físicos. Assim, a análise estrutural é a determinação dos efeitos das cargas nas estruturas físicas e seus componentes.

Sobre os elementos estruturais é preciso ter conhecimento, por exemplo, do material do qual ele é composto, como madeira, concreto, aço, ferro, dentre outros. A engenharia estrutural depende de conhecer esses materiais e suas propriedades, a fim de entender como diferentes materiais suportam e resistem às cargas [Soriano, 2013].

Os materiais foram modelados no EngPack Analysis em suas classes do componente Modelo. Uma vez que o requisito dimensional para uma estrutura tenha sido definido, torna-se necessário determinar as cargas que a estrutura deve suportar. $\mathrm{O}$ projeto estrutural, começa com a especificação de cargas que atuam na estrutura. Para tanto, o sistema foi desenvolvido para modelar os elementos estruturais desenhados no ambiente CAD em classes bem definidas (Material, Seção, Elemento, Nó, Carregamento, etc.), de forma que essas possam ser convertidas em matrizes, com o intuito da utilização dessas matrizes para a realização do cálculo de análise, em forma de rotina computacional. Essas análises resultam, dentro do EngPack Analysis, em diagramas de cisalhamento (força cortante) e momento fletor em um determinado ponto de um elemento estrutural.

\section{Desenvolvimento do Sistema}

O EngPack Analysis provê um conjunto de ferramentas que possibilita a modelagem de estruturas planas, do tipo vigas, pórticos e treliças, que funcionam no ambiente CAD do sistema, onde é possível desenhar os elementos que compõem a estrutura. Essa modelagem consiste na inserção, remoção e edição de elementos de barra na área de 
desenho CAD e a aplicação de informações de materiais, seções, condições de apoio, forças e carregamentos aos componentes da estrutura. Além disso, é possível adicionar novos componentes, como rotinas de análise, de modo que estas possam ser executadas sobre uma determinada estrutura.

É possível a criação de múltiplas estruturas em um único arquivo de projeto, possibilitando a criação, abertura e salvamento desses arquivos, além da exportação de relatórios sobre os cálculos realizados pelas rotinas implantadas. Ainda provê suporte aos recursos fundamentais em um sistema de edição, como a cópia, recorte e colagem de elementos por meio da área de transferência, sendo possível desfazer e refazer ações.

Com uma estrutura modelada no sistema, é possível efetuar a análise das reações e esforços resultantes das forças e carregamentos aplicados sobre os elementos da estrutura. Essa análise gera diagramas que são exibidos no próprio ambiente CAD. Soriano [2013] destaca que os diagramas podem ser usados para determinar facilmente o tipo, tamanho e material de um elemento em uma estrutura, de modo que um determinado conjunto de cargas possa ser suportado sem falha estrutural.

O componente Modelo lida com os dados do sistema, mais especificamente os das estruturas e projetos manipulados, além de armazenar e encapsular informações das matrizes necessárias para análise e dos sistemas de arquivos. Como trata-se de um sistema feito em uma linguagem orientada à objetos, o componente Modelo contém as classes que representam as estruturas com as quais o sistema lida, sendo elas: Node, Member, Material, Section, NodeForce, MemberLoad, Structure, Project, entre outras.

$\mathrm{Na}$ implementação foi considerado que um projeto pode possuir diversas Estruturas, que são compostas por Nós e Elementos. Os Nós da estrutura podem ter uma Força, significando que a Força está aplicada sobre ele, assim como um Elemento pode ter um Carregamento aplicado a ele. Material e Seção podem ser definidos também.

As classes do componente Modelo são manipuladas pelas classes do componente Controlador, sendo essas classes responsáveis pela comunicação da interface gráfica com o modelo de negócio. O componente Controlador é responsável pela manipulação dos arquivos de projetos modelados no componente Modelo e pela efetiva análise das estruturas modeladas, realizando os cálculos para obtenção dos esforços e reações na estrutura, estando nesse componente as rotinas de análise. Dentre as principais classes do componente Controlador, destacam-se: Diagram, responsável pela transformação dos resultados das análises em diagramas na interface gráfica do usuário; BeamAnalysis, faz a análise de esforço em vigas [Filho, 2018]; TrussFrameAnalysis, responsável pela análise de treliças e pórticos planos [Filho, 2018]. Nesse componente há ainda classes responsáveis pelo gerenciamento de arquivos de projeto, relatórios e classes que controlam cada painel de ferramentas do programa.

O componente Visão possui basicamente classes relacionadas à cada componente gráfico da interface, sendo responsáveis por obter as informações da interação com o usuário e enviá-las para as classes do componente controlador, que retornará algum tipo de informação a ser repassada graficamente para o usuário. Nesse componente estão as classes que representam o ambiente de desenho CAD do sistema.

Todas as classes são organizadas em pacotes de acordo com suas finalidades, se comunicando por meio do componente central da interface gráfica, o AnalysisUI. O padrão Composite é utilizado nas classes que compõem o pacote que permite a 
implementação de novos componentes e módulos, como no funcionamento da hierarquia dos componentes de interface gráfica. Algumas das classes controladoras da interface gráfica especificam a classe Controller por meio de herança e são ainda constituídas por objetos dessa classe.

Outro padrão de projeto utilizado na implementação é o Observer que é usado em quase todas as informações das classes do componente Modelo do sistema. Um exemplo é nos atributos da classe Section, que ao serem alterados notificam os observadores da interface. As informações contidas na classe Section, ao serem alteradas, devem notificar os observadores presentes em diferentes partes da interface, como no painel de manipulação de seções e no de estrutura de projeto. Um padrão também amplamente aplicado na implementação do sistema, principalmente para o controle das ações na interface gráfica, foi o padrão Command, sendo que parte das operações possíveis de serem executadas pelo usuário estão encapsuladas por meio desse padrão, como desfazer ações, adição e remoção de elementos no ambiente CAD. No pacote Command estão as classes abstratas Command e CommandGroup. Qualquer comando que possa ser manipulado pelo usuário deve especificar essas classes, como os comandos de adição, remoção e movimentação de componentes na classe StructureGraphic ou os comandos de manipulação da área de transferência na classe ClipBoard. Esses comandos podem ser agrupados, através da classe CommandGroup.

\subsection{Interfaces para Desenvolvimento de Componentes}

Além dos componentes descritos, o sistema possui funcionalidades que possibilitam a criação de novos módulos e componentes. Essa possibilidade se dá por meio de classes e interfaces que permitem a fácil implementação de diferentes itens de interface gráfica para serem acoplados à módulos existentes ou ainda a novos módulos criados. Assim, é possível expandir o sistema para suportar futuras implementações de rotinas, bastando comunicar essas rotinas com os novos componentes, como exemplo o dimensionamento de estruturas, que vem sendo desenvolvida no projeto que esta pesquisa está inserida.

No pacote Module desse componente, a classe abstrata Module descreve o modelo básico para criação de um módulo (o EngPack é um projeto maior, dessa forma o EngPack Analysis é um módulo desse sistema). A classe abstrata ModuleController descreve as operações básicas para o componente que deve controlar a interface gráfica do módulo, como adicionar e remover componentes da interface. Na Figura 1 é apresentada a tela do EngPack Analysis, em um projeto aberto com algumas informações.

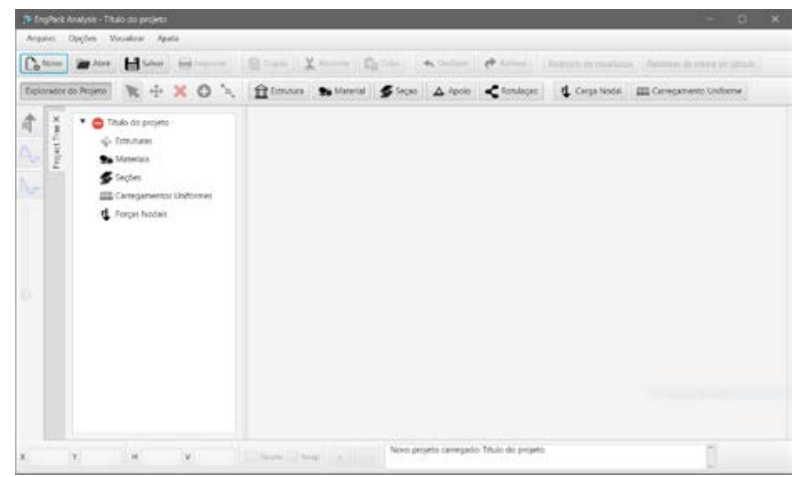

Figura 1. Tela principal com projeto aberto 
Modelo de Dados: O EngPack Analysis possui um tipo de arquivo padrão que é manipulado por ele, esse arquivo representa um projeto de estruturas. Um projeto no sistema pode ser visto como um conjunto de listas de objetos e cada objeto pode estar relacionado a outros da mesma lista ou de listas diferentes. Os arquivos de projetos gerenciados pelo sistema armazenam todas as informações do projeto, como a lista de estruturas com suas informações, que incluem listas de nós, elementos, materiais, seções, forças e carregamentos.

Interface Gráfica do Usuário: A organização da interface segue um padrão de barras e painéis, como mostrado na Figura 1, comum em muitos programas de análise ou mesmo em sistemas CAD. No topo da tela há uma barra de menu com acesso às principais ferramentas/funcionalidades do sistema. Na parte de baixo têm-se as barras de ferramentas do sistema, uma barra para ferramentas gerais de edição e uma barra com ferramentas específicas de modelagem e análise de estruturas. Ainda há uma barra de ferramentas de diagramas do lado esquerdo da tela, a qual permite a exibição dos diagramas gerados, há também um painel que contém a estrutura do projeto, composto pelas listas de componentes do mesmo. Ao centro há o ambiente de desenho CAD para cada estrutura aberta e por fim, no rodapé há uma barra de status com informações de coordenadas do ambiente CAD aberto. O restante das ferramentas está distribuído em painéis flutuantes. Por exemplo, há painéis para gerenciamento de materiais, seções, carregamentos, entre outras funcionalidades do sistema. Além disso, esses painéis podem ser ancorados em algum dos lados na janela, possibilitando personalizar o ambiente de edição. Cada componente da interface possui uma classe controladora. Esses componentes são modularizados, de modo que cada um é uma parte separada do componente Visão do sistema.

\section{Discussões e Resultados}

Uma etapa fundamental no processo de desenvolvimento de software é a avaliação do sistema, que pode ser feita utilizando diferentes métodos conhecidos na literatura. Uma das formas de se verificar a usabilidade é a aplicação de Estudos de Caso, onde diferentes situações de uso do sistema são testadas por usuários afim de obter informações, como o tempo para efetuar uma operação, a quantidade de cliques do mouse para executá-la ou, ainda, questões sobre a agradabilidade do sistema.

Seguindo orientações definidas em YIN [2008], desenvolver um estudo com a qualidade desejada requer utilizar usuários reais do sistema, para coletar dados, problemas e futuras melhorias. Assim, para avaliação do presente sistema utilizou-se um Estudo de Caso para coletar as impressões sobre a utilização do mesmo, por meio da aplicação de um questionário. Para tanto, teve-se como público-alvo os alunos e professores do curso de EC, da UFCAT, sendo os potenciais usuários finais do sistema. Após a realização do estudo, os resultados foram documentados e analisados, com o intuito dos dados refletirem em melhorias ao EngPack Analysis.

\subsection{Aplicação do Estudo de Caso}

O Estudo de Caso foi feito em duas etapas. A primeira envolveu a utilização do EngPack Analysis para análise de vigas, através de um passo-a-passo guiado por 3 professores de disciplinas do curso de EC, que envolvam a utilização de sistemas de análise de estruturas. O sistema foi apresentado aos participantes sem treinamento prévio, apenas com orientações dadas pelo aplicador do estudo. A finalidade foi 
observar como o participante, sendo usuário experiente, se comporta frente a um ambiente de sistema CAD, evidenciando as ações mais comuns tomadas.

Por meio da exposição da opinião dos participantes, os resultados foram coletados e anotados para análise. Esses revelaram a ausência de itens na interface gráfica que proporcionassem uma melhor percepção pelo usuário a respeito, por exemplo, das medidas dos elementos que eram inseridos no ambiente. Além disso, mostrou-se a necessidade da simplificação de certos processos de modelagem, como o de criação e aplicação de componentes como materiais, seções e carregamentos.

A segunda etapa foi mais abrangente e formal, com o sistema tendo sofrido alterações para a correção dos erros (evidenciadas na primeira etapa). Como apoio foram disponibilizados o sistema e um manual básico de sua utilização, com explicações a respeito do funcionamento da interface gráfica. Foi entregue uma rotina de testes a ser realizada, onde estavam descritas as tarefas padronizadas, como a inserção de estruturas do tipo viga e um questionário de usabilidade foi respondido. Esta etapa foi realizada em sala de aula e contou com a participação de 12 (doze) voluntários (alunos da disciplina de análise estrutural), sendo estes observados pelo aplicador do estudo enquanto realizavam tarefas simples orientadas.

A interação do usuário foi medida pelo próprio sistema, com o objetivo de observar possíveis erros cometidos pelos usuários na execução de tarefas. Os mapas de calor são apresentados na Figura 2. Com essas informações foi possível perceber a preferência do usuário pelo posicionamento de elementos na interface.

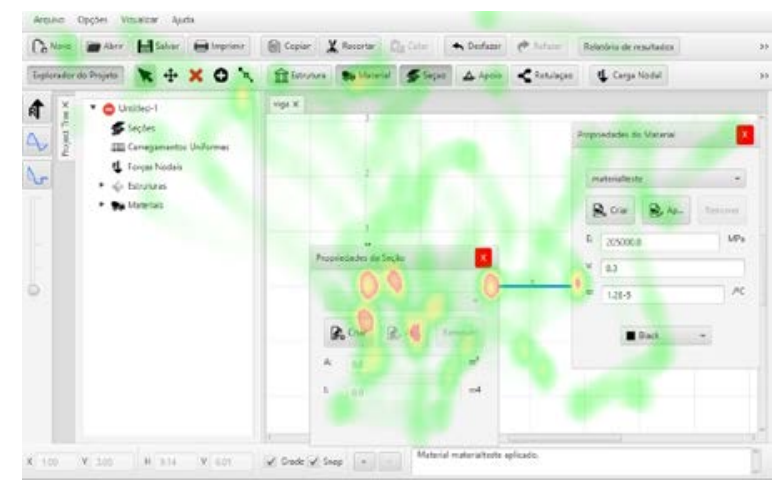

Figura 2. Exemplo de Mapa de Calor

Associados aos mapas de calor, foram coletados os logs de execução das tarefas, que trazem informações como o horário de execução da tarefa, quantidade de cliques e tempo gasto para a execução. Esses dados ajudam a rastrear possíveis erros na interação do participante com o sistema, bem como suas dificuldades na utilização do mesmo. Todas as informações coletadas foram enviadas de forma anônima para um sistema de arquivos, onde foram analisados os resultados concretos para se ter conhecimento sobre o uso do sistema pelos participantes. Apenas informações do próprio sistema foram capturadas.

No final da execução do Estudo de Caso, após a realização dos testes, foi solicitado aos participantes responderem um questionário, afim de coletar dados sobre a utilização do sistema. De acordo com Sharp, Rogers e Preece [2007] os questionários são uma técnica bem estabelecida de coleta de dados e de opiniões, podendo conter perguntas abertas ou fechadas, afim de aprofundar entendimentos. 


\subsection{Respostas às Questões}

Nesta seção são apresentadas as perguntas e as respostas dos participantes que participaram do Estudo de caso.

Q1. Quais dos programas abaixo você já utilizou para realizar análises em estruturas? $O$ intuito aqui foi verificar quais sistemas de análise estrutural os participantes já haviam utilizado, para então identificar qual tipo de ambiente de análise eles estavam familiarizados. Todos os participantes responderam que já utilizaram o FTOOL para análise de estruturas, 2 (16,7\%) também usaram Ansys e 1 (8,3\%) respondeu SAP e ATRAP. Esse resultado indica que eles, possivelmente, utilizaram o sistema de forma parecida como utilizariam o FTOOL, sendo que a diferença entre os sistemas poderia indicar certa resistência na utilização do EngPack Analysis.

Q2. Há quanto tempo você utiliza os programas marcados na questão anterior? A maioria dos participantes já utilizam o sistema FTOOL há mais de 1 ano, sendo 75\% destes, possuindo um nível relevante de conhecimento sobre a utilização desse sistema. Tal informação torna o processo de aprendizado do EngPack Analysis mais simples. $16,7 \%$ usam entre 6 meses e abaixo de 1 ano e 8,3\% há menos de 6 meses.

Q3. Como você considera seu nível de experiência em relação à utilização dos programas mencionados anteriormente? (peso 1 pouco experiente ao peso 5 muito experiente)? É possível observar que a maioria se considera experiente na utilização de sistemas de análise de estruturas como o FTOOL, tendo 6 (50\%) participantes respondido com peso 4, 4 (33,3\%) com peso 3 e 2 (16,7\%) peso 2. Esse resultado é relevante para validação deste Estudo de Caso, visto que os participantes têm o conhecimento para avaliar um sistema como o EngPack Analysis, uma vez que metade possui habilidades com outro sistema do mesmo tipo.

Após a seção de perguntas sobre a experiência dos usuários, seguiu-se com perguntas sobre a facilidade de utilização dos diversos recursos do EngPack Analysis. Essas questões utilizam-se da escala likert para medição da usabilidade, com escala numérica de 1 a 5 (onde 1 significa muito fácil e 5 muito difícil).

Q4. Qual o nível de facilidade na criação de um novo projeto? A maioria, 8 participantes (66,7\%), responderam 1, 2 (16,7\%) escolherem 2 na escala e 1 (8,3\%) nota 3 e nota 5, revelando que o processo de criação de um novo projeto é considerado simples e fácil de ser executado.

Q5. Qual o nível de facilidade na criação de uma nova estrutura? A maioria dos participantes, 8 participantes $(66,7 \%)$, consideram que esse processo é relativamente fácil, deram nota 1, $2(16,7 \%)$ nota 2 e $1(8,3)$ nota 3 e nota 5.

Q6. Qual o nível de facilidade na inserção, remoção e edição de elementos? O processo de modelagem de uma estrutura foi considerado com um baixo grau de dificuldade por 6 participantes, sendo que 5 (41,7\%) deram nota 2 e 1 deu nota 4 . Esse resultado confirma a necessidade da simplificação, que foi alterado por meio de ferramentas mais fáceis e claras de serem utilizadas.

Q7. Qual o nível de facilidade de navegação no ambiente CAD do EngPack Analysis (mover visualização, aplicar zoom, selecionar componentes)? Um dos requisitos mais críticos do sistema desenvolvido foi o ambiente CAD, levando em 
consideração a necessidade de torná-lo o mais fluído possível, uma vez que essa característica deveria transparecer na forma como o usuário poderia navegar na área de desenho desse ambiente. As respostas revelam que esse requisito foi cumprido parcialmente, visto que 7 participantes (58,3\%) consideraram muito fácil. 2 (16,7\%) deram nota 3 e 4, sendo uma boa média e 1 deu nota 5, considerando muito difícil.

As questões das funcionalidades permitiam sugestões de modificações ou relatos de problemas identificados, que foram analisados e solucionados. Como exemplo temse a sugestão de alteração da cor do componente gráfico que indica a origem do plano cartesiano do ambiente CAD, de modo que não atrapalhasse na inserção dos elementos. Assim, para melhoria fez-se a alteração desse componente tornando sua cor desbotada, reduzindo o contraste para os demais elementos gráficos, conforme o princípio de Kimball [2013]: Constraste, Repetição, Alinhamento e Proximidade (CRAP).

Ainda, alguns usuários relataram problemas na utilização do sistema em laptops sem mouse, ou seja, usuários que tinham disponível apenas o touchpad do seu computador. Esses problemas estão relacionados às ferramentas de pan (mover a visualização) e zoom (aproximar ou afastar a visualização) do ambiente CAD que são utilizadas por meio do botão de rolagem do mouse. A implementação da correção está como trabalhos futuros.

A parte final do questionário está relacionada às questões gerais em relação a usabilidade e agradabilidade da interface gráfica do sistema, com uma escala numérica de 1 a 5 (onde 1 representa total discordância e 5 total concordância).

Q8. Os botões, na interface gráfica, eram claros e precisos sobre sua funcionalidade? A análise das respostas foi satisfatória, uma vez que 3 (25\%) dos participantes derem valor 5 e outros 3 valor 4, indicando que os botões conseguem representar sua funcionalidade. Em contrapartida, a outra metade demonstrou insatisfação, onde 1 participante deu nota 3, 2 deram nota 2 e 3 (25\%) nota 1. Isso demonstra a necessidade de organizar os ícones e botões de forma que esses possam expressar melhor sua funcionalidade. Ações foram tomadas para minimizar o problema identificado, alterando os símbolos dos botões para que estes se tornassem mais claros e objetivos. Além disso, houve acréscimo de texto nos botões que possuíam apenas símbolos, de modo que o usuário tenha mais informações acerca da funcionalidade.

Q9. O tamanho dos textos estava adequado para visualização/leitura? Para a maioria dos participantes, 7 (58,3\%), o tamanho da fonte dos textos estava adequado para visualização, uma vez que esses deram nota 5, referindo-se a total concordância. Entretanto, 3 (25\%) deram nota 4 e $2(16,7 \%)$ nota 1. Para tanto, uma nova funcionalidade para opção de ajuste de tamanho de texto foi implementada e disponibilizada. É importante ressaltar que essa estratégia contempla aspectos de acessibilidade, além da usabilidade.

Q10. Prefiro cores mais escuras a cores mais claras? 4 (33,3\%) dos participantes deram nota 5, referindo-se a total concordância, 3 (25\%) nota 4 e 4 participantes deram nota 3. Apenas $1(8,3 \%)$ deu nota 1 , sugerindo total discordância. Assim, foi implementada uma funcionalidade que possibilite trocar as cores do ambiente CAD do sistema e, posteriormente, a implementação dessa possibilidade para todo o sistema pode ser efetuada em trabalhos futuros. 
Q11. Tive maior facilidade ao trabalhar com o EngPack Analysis em comparação ao FTOOL? Esta questão teve por intuito comparar a utilização entre os sistemas, sendo o FTOOL uma das ferramentas mais utilizadas no meio acadêmico para análise de estruturas. As respostas evidenciaram equilíbrio na opinião dos participantes, uma vez que parte das respostas consideraram que o EngPack Analysis tem utilização mais fácil e a outra parte respondeu de forma neutra. Onde 4 (33,3\%) dos participantes deram nota 5, 3 (25\%) nota 4 e nota 3 e 2 (16,7\%) nota 2.

Os resultados coletados pelo questionário, realizado nesta segunda etapa do Estudo de Caso, foram fundamentais para validar os componentes da interface gráfica do sistema desenvolvido, mostrando que este possui interface gráfica de fácil utilização e aprendizado. Entretanto, alguns desses componentes necessitaram de pequenas alterações para correta validação, como mencionado anteriormente. Ainda, observou-se que o EngPack Analysis consegue realizar a análise das estruturas conforme proposto neste trabalho.

\section{Conclusões}

Neste artigo está descrita uma pesquisa que ressalta a empregabilidade da interdisciplinaridade entre as áreas de ES com a EC, uma vez que os sistemas computacionais são de grande aceitação e necessidade em várias áreas de conhecimento.

Como previamente discutido, esta pesquisa teve como motivação a demanda pela utilização de um sistema de análise de estruturas pelos estudantes e professores do departamento de EC da UFCAT de modo gratuito, que comtemple as reais necessidades das disciplinas empregadas no curso.

Técnicas da ES foram utilizadas para estruturar o desenvolvimento desse sistema, fundamentadas em suas metodologias de desenvolvimento de software, bem como, fazendo uso de ferramentas para codificação e testes. Ainda, para desenvolvimento do EngPack Analysis foi estabelecido que ele seguisse padrões utilizados por sistemas no mesmo domínio, com vistas a reduzir a curva de aprendizado dos usuários. Assim, o tempo no processo de adaptação do EngPack Analysis foi reduzido, trazendo maior aceitabilidade, além dos benefícios apresentados.

Com a aplicação do Estudo de Caso observou-se que o sistema é capaz de realizar as análises propostas, porém verificou-se também a necessidade de melhorias, como o aprimoramento da interface, relacionado a usabilidade e acessibilidade.

Durante o processo de desenvolvimento do EngPack Analysis, iniciou-se também o desenvolvimento de sua versão Web, sendo este trabalho futuro. Ainda, temse como meta finalizar a implementação da transição para um ambiente de desenho tridimensional e avaliar o mesmo para uma população maior de usuários, com foco no mercado de trabalho.

Por fim, espera-se que este trabalho sirva como base de conhecimento para novas pesquisas envolvendo as áreas de estudo contempladas, e que inspire projetos interdisciplinares para que possam ser desenvolvidos com vistas a contemplar problemas relacionados ao mercado e à comunidade acadêmica. 


\section{Referências}

Sarcar, M. M. M, Rao, K. M. e Narayan, K. L. Computer aided design and manufacturing. second. PHI Learning Pvt. Ltd., 2008.

Korpyljov, D. e Tkachenko, S. Development and research of distributed web-oriented architectures cad design engineering. 12th International Conference on the Experience of Designing and Application of CAD Systems in Microelectronics (CADSM). 2013. p. 453-454.

Bizello, S. e Ruschel, R. Estudo de CAD livre para implementação de ferramenta de projeto. Em: Gestão \& Tecnologia de Projetos, v. 6, n. 1, p. 32-52, 2011.

Sommerville, I. Software Engineering. 10th. ed. USA: Addison-Wesley Publishing Company, 2019. ISBN 978-8543024974, 768 páginas.

Lobato, L. L. An approach for Risk Management in Software Product Lines. Tese (Ph.D. Thesis) - Federal University of Pernambuco, Recife - Brazil, 2012.

Dutra, R. V. Software para análise matricial de esforços em estruturas de vigas planas. 2018. TCC (Graduação), Departamento de Engenharia Civil, Faculdade de Engenharia, Universidade Federal de Catalão, Catalão.

Martha, L. F. FTOOL: Um Programa Gráfico-Interativo para Ensino de Comportamento de Estruturas. 2012.

Filho, J. M. da S. Software para análise estrutural de treliças planas. 2018. TCC (Graduação), Departamento de Engenharia Civil, Faculdade de Engenharia, Universidade Federal de Catalão, Catalão.

Longo, L. F. Desenvolvimento de um aplicativo de análise de estruturas reticuladas planas em plataforma Android. 2015. TCC (Graduação), Departamento de Engenharia Civil, Universidade Federal de Santa Catarina.

Viegas, M. A. e Vargas, A. Desenvolvimento de software para simulação e solução de esforços em vigas como ferramenta educacional. Engenharia Civil da UNESC, 2015.

Kaefer, L. F. Desenvolvimento de uma ferramenta gráfica para análise de pórticos de concreto armado. Dissertação. Escola Politécnica, USP, São Paulo, 2000.

Luciano, J. e Alves, W. J. B. Padrão de arquitetura MVC: Model-view-controller. EPeQ Fafibe, v. 01, n. 3a. Ed., p. 102-107, 2011.

Gamma, E., Helm, R., Johnson, R. e Vlissides, J. Design Patterns: Elements of Reusable Object-oriented Software. USA: Addison-Wesley Longman Publishing Co. 1995.

Soriano, H. L. Estática das Estruturas. Third edition: Ciência Moderna, 2013.

Yin, R. K. Case Study Research: Design and Methods (Applied Social Research Methods). Fourth edition. [S.l.]: Sage Publications, 2008. ISBN 1412960991.

Sharp, H., Rogers, Y. e Preece, J. Interaction Design: Beyond Human Computer Interaction. USA: John Wiley \& Sons, Inc., 2007. ISBN 0470018666.

Kimball, M. A. Visual design principles: An empirical study of design lore. Journal of Technical Writing and Communication, v. 43, n. 1, p. 3-41, 2013. 